\title{
Use of nebulised magnesium sulphate as an adjuvant in the treatment of acute exacerbations of COPD in adults: a randomised double-blind placebo-controlled trial
}

\author{
Llifon Edwards, ${ }^{1,2}$ Philippa Shirtcliffe, ${ }^{1,2}$ Kirsten Wadsworth, ${ }^{1,3}$ Bridget Healy, 1,2 \\ Sarah Jefferies, ${ }^{1,2}$ Mark Weatherall, ${ }^{2,4}$ Richard Beasley, ${ }^{1,2}$ on behalf of the \\ Magnesium COPD Study Team
}

\begin{abstract}
${ }^{1}$ Medical Research Institute of New Zealand, Wellington, New Zealand

${ }^{2}$ Capital and Coast District Health Board, Wellington, New Zealand ${ }^{3}$ Hutt Valley District Health Board, Lower Hutt, New Zealand

${ }^{4}$ Wellington School of Medicine and Health Sciences, Wellington, New Zealand
\end{abstract}

Correspondence to Dr Philippa Shirtcliffe, Medical Research Institute of New Zealand, P O Box 7902, Wellington 6242

New Zealand; pip.shirtcliffe@mrinz.ac.nz

Received 31 May 2012 Revised 21 November 2012 Accepted 29 November 2012 Published Online First 8 January 2013
To cite: Edwards $L$ Shircliffe $P$, Wadsworth $K$, et al. Thorax 2013:68: 338-343.

\begin{abstract}
Background Intravenous magnesium has been shown to cause bronchodilation in acute severe asthma and in small trials in acute exacerbations of chronic obstructive pulmonary disease (AECOPD). There is also some evidence of benefit from nebulised magnesium in acute severe asthma. Our hypothesis was that adjuvant magnesium treatment administered via repeated nebulisation was effective in the management of AECOPD.

Methods In this randomised double-blind placebocontrolled trial, we approached 161 patients with AECOPD presenting to the emergency departments at two New Zealand hospitals with a forced expiratory volume in $1 \mathrm{~s}\left(\mathrm{FEV}_{1}\right)<50 \%$ predicted 20 min after initial administration of salbutamol $2.5 \mathrm{mg}$ and ipratropium $500 \mu \mathrm{g}$ via nebulisation. Patients received $2.5 \mathrm{mg}$ salbutamol mixed with either $2.5 \mathrm{ml}$ isotonic magnesium sulphate (151 mg per dose) or $2.5 \mathrm{ml}$ isotonic saline (placebo) on three occasions at 30 min intervals via nebuliser. The primary outcome measure was $\mathrm{FEV}_{1}$ at 90 min.
\end{abstract}

Results 116 patients were randomised, 52 of whom were randomly allocated to the magnesium adjuvant group. At 90 min the mean (SD) FEV 1 in the magnesium group $(\mathrm{N}=47)$ was $0.78(0.33)$ I compared with 0.81 $(0.30)$ I in the saline group $(\mathrm{N}=61)$ (difference -0.026 I $(95 \% \mathrm{Cl}-0.15$ to $0.095, \mathrm{p}=0.67)$. No patients required non-invasive ventilation. There were $43 / 48$ admissions to hospital in the magnesium group and 56/61 in the saline group (RR $0.98,95 \% \mathrm{Cl} 0.86$ to $1.10, p=0.69$ ).

Conclusions Nebulised magnesium as an adjuvant to salbutamol treatment in the setting of AECOPD has no effect on $\mathrm{FEV}_{1}$.

Australian New Zealand Clinical Trials Registry ACTRN12608000167369.

\section{INTRODUCTION}

Magnesium has a key role in numerous physiological processes. ${ }^{1}{ }^{2}$ Important underlying mechanisms of action of magnesium include calcium antagonism via calcium channels, regulation of energy transfer (such as the production and function of ATP) and membrane stabilisation. ${ }^{12}$ In the airways, magnesium is a bronchodilator through various mechanisms including an inhibitory effect on bronchial smooth muscle

\section{Key messages}

What is the key question?

- Is there a role for nebulised magnesium treatment in the management of acute exacerbations of chronic obstructive pulmonary disease (AECOPD)?

What is the bottom line?

- Both single and repeated doses of nebulised magnesium, as an adjunct to nebulised salbutamol treatment in the setting of $A E C O P D$, have no effect on $\mathrm{FEV}_{1}$.

\section{Why read on?}

- Intravenous or nebulised magnesium is attractive as a bronchodilator because it is relatively cheap and has minimal side effects. There is an ongoing interest in a possible therapeutic role for intravenous or nebulised magnesium in obstructive airways disease. The result of this randomised double-blind placebo-controlled trial in AECOPD is discussed in the context of available evidence.

contraction mediated by calcium ${ }^{3}$ and an inhibitory effect on acetylcholine release from cholinergic nerve terminals ${ }^{4}$ and histamine from mast cells. ${ }^{3}$

Experimental studies have variably demonstrated a benefit of magnesium in acute severe asthma since the first report over 50 years ago. ${ }^{5}$ Magnesium has been administered via the intravenous route ${ }^{6-10}$ and via nebuliser, ${ }^{11-14}$ with interest in the latter route of administration because of practical and potential safety advantages. In a randomised double-blind placebocontrolled trial, we have previously shown that isotonic nebulised magnesium sulphate results in an enhanced bronchodilator response in severe exacerbations of asthma. ${ }^{13}$ However, fewer studies have addressed the effects of magnesium in chronic obstructive pulmonary disease (COPD) ${ }^{15-20}$ even though asthma and COPD share some pathophysiological characteristics (such as bronchial hyper-responsiveness) as well as numerous therapies, particularly bronchodilator treatments. 
We are aware of reports of only six studies investigating the bronchodilator efficacy of magnesium sulphate in COPD. Three studies have reported the efficacy of intravenous magnesium in the setting of acute exacerbations of chronic obstructive pulmonary disease (AECOPD), improving patient symptoms and reduced length of stay, ${ }^{15}$ increasing peak expiratory flow ${ }^{16}$ and increasing forced expiratory volume in $1 \mathrm{~s}\left(\mathrm{FEV}_{1}\right) .{ }^{19}$ This last study only showed an effect as an adjunct to inhaled $\beta_{2}$ agonists. A fourth study combined one intravenous bolus of magnesium with repeated nebulised magnesium and terbutaline. There was no difference in the primary combined outcome of hospital admission, intubation and hospital death rate compared with terbutaline and ipratropium. ${ }^{20}$ However, the terbutaline and ipratropium group showed a greater improvement in peak expiratory flow. Two studies in stable COPD have been undertaken, reporting a reduction in hyperinflation with intravenous magnesium ${ }^{17}$ and an increase in $\mathrm{FEV}_{1}$ when magnesium was added to nebulised salbutamol. ${ }^{18}$

Nebulised magnesium is attractive as a therapeutic option because it is easily administered, relatively cheap and has minimal side effects. In light of some evidence for an effect when nebulised in severe exacerbations of asthma, the similarities between asthma and COPD (especially with regard to bronchodilator therapy) and the practical advantages of administration via nebuliser, we sought to focus on the nebulised route of delivery in AECOPD. Our hypothesis was that adjuvant magnesium treatment administered via nebuliser was effective in the management of patients with AECOPD.

\section{METHODS}

\section{Participants}

Patients with an AECOPD who presented to the emergency departments (EDs) of two university hospitals in New Zealand (Wellington Regional Hospital and Hutt Hospital) were invited to participate in the study between June 2008 and November 2011, if a study investigator was available. Inclusion in the study required age $\geq 35$ years, a doctor diagnosis of COPD, ratio of the $\mathrm{FEV}_{1}$ to forced vital capacity (FVC) $\left(\mathrm{FEV}_{1} / \mathrm{FVC}\right)<70 \%$ and an $\mathrm{FEV}_{1} \leq 50 \%$ predicted $20 \mathrm{~min}$ after initial treatment with $2.5 \mathrm{mg}$ salbutamol and $500 \mu \mathrm{g}$ ipratropium bromide by nebulisation. We excluded patients if they required intubation or noninvasive ventilation (NIV), were unable to perform spirometry or had evidence of pneumothorax, hypotension, any other serious medical condition that would prevent their participation in the trial or were pregnant.

\section{Study protocol}

On presentation to the ED with a provisional diagnosis of an AECOPD, potential subjects were clinically assessed and received standard initial treatment (ie, $2.5 \mathrm{mg}$ salbutamol and $500 \mu \mathrm{g}$ ipratropium bromide by jet nebulisation and $40 \mathrm{mg}$ prednisone). Oxygen ( $2 \mathrm{l} / \mathrm{min}$ nasal prongs) was given if oxygen saturations on room air were $<90 \%$. Only subjects with an $\mathrm{FEV}_{1} \leq 50 \%$ predicted measured $20 \mathrm{~min}$ after commencement of the initial salbutamol/ipratropium nebulisation were enrolled in the trial. During this $20 \mathrm{~min}$ period informed consent was obtained and a brief questionnaire administered, obtaining information with regard to duration and severity of symptoms, medication use and smoking status. Routine blood tests (creatinine, sodium, potassium, full blood count) were done, as well as a serum magnesium level. After randomisation, patients received by jet nebulisation $2.5 \mathrm{mg}$ salbutamol (GlaxoSmithKline, London, UK) mixed with $2.5 \mathrm{ml}$ isotonic magnesium sulphate ( $250 \mathrm{mmol} / \mathrm{l}$, tonicity $289 \mathrm{mosmol} ; 151 \mathrm{mg}$ per dose) or $2.5 \mathrm{ml}$ isotonic saline (placebo) on three occasions at 30 min intervals. The majority of nebulisers were driven by air, 14 patients received supplemental oxygen via nasal prongs during the nebuliser $(1-2 \mathrm{l} / \mathrm{min})$ and 21 had their nebuliser driven by oxygen (6 l/min), most commonly because medical air was not available.

We recorded $\mathrm{FEV}_{1}$ (and FVC) using a hand-held spirometer (Micro Medical, Rochester, Kent, UK) on first referral ('presentation' $\mathrm{FEV}_{1}$ ), before the first study nebuliser ('baseline' $\mathrm{FEV}_{1}$ ), before each subsequent nebulisation and then $30 \mathrm{~min}$ after the last nebule. Three measurements were made at each time point and the highest recording used for analysis. All those performing spirometry received training from a respiratory scientist. Pulse oximetry was done as part of routine clinical observations (blood pressure, pulse, respiratory rate) and otherwise as clinically indicated. After the final recordings the decision to admit the patients was made at the discretion of the clinical team.

\section{Randomisation and masking}

Patients were randomly allocated in a double-blind fashion to receive one of two treatment regimens. The study statistician performed block randomisation with a block size of eight using a computer-generated random sequence. This was administered by a third-party process so that participants and investigators were unaware of treatment allocation through provision by the hospital pharmacy of pre-prepared identical syringes containing the study drug or placebo according to this random allocation.

\section{Statistical analysis}

Analysis was by intention-to-treat. The primary outcome was $\mathrm{FEV}_{1}$ at $90 \mathrm{~min}$. Secondary outcomes were $\mathrm{FEV}_{1}$ at 30 and $60 \mathrm{~min}$, hospital admission, episodes of NIV and admission to ICU.

The significance level was set at $p=0.05$. The primary analysis was a t test comparing $\mathrm{FEV}_{1}$ between the randomised groups at $90 \mathrm{~min}$. Secondary analyses were $t$ tests to compare $\mathrm{FEV}_{1}$ at 30 and $60 \mathrm{~min}$ as well as ANCOVA with adjustment for baseline $\mathrm{FEV}_{1}$. The calculation of relative risk of the secondary categorical outcome measurements with appropriate CIs was planned but, in the event, there were no episodes of NIV or ICU admissions and this could only be calculated for hospital admissions. An exploratory analysis of the relationship between the change in $\mathrm{FEV}_{1}$ and serum magnesium was carried out using simple correlation coefficients and ANCOVA. SAS V.9.2 (SAS Institute, Cary, North Carolina, USA) was used for the analysis.

The planned sample size of 200 participants was estimated based on the SD of the $\mathrm{FEV}_{1}$ at the last measurement time from our previous study. ${ }^{13}$ In that study, the difference between the mean $\mathrm{FEV}_{1}$ in the magnesium group (1.94 l) and in the saline group $(1.581)$ was 0.361 with a pooled SD of 0.741 . To detect an absolute difference in $\mathrm{FEV}_{1}$ of $0.30 \mathrm{l}$ at an $\alpha$ of $5 \%$ and a power of $80 \%$ required 194 subjects in a two-arm trial.

\section{RESULTS}

One hundred and sixty one patients were assessed between May 2008 and December 2011. Following exclusion of 45 patients, 116 patients were randomised (figure 1). The reasons for exclusion included not meeting the inclusion criteria (lack of formal COPD diagnosis, significant other comorbidity such as pneumonia and congestive heart failure, receiving NIV on arrival at the $\mathrm{ED}, \mathrm{FEV}_{1}>50 \%$ predicted post-bronchodilator), declined to participate and other (eg, unable to consent secondary to language barrier or dementia, unable to perform adequate spirometry, given intravenous magnesium by ED staff).

Of the 116 remaining patients, 52 were randomly allocated to the magnesium adjuvant group. Two patients in the placebo 


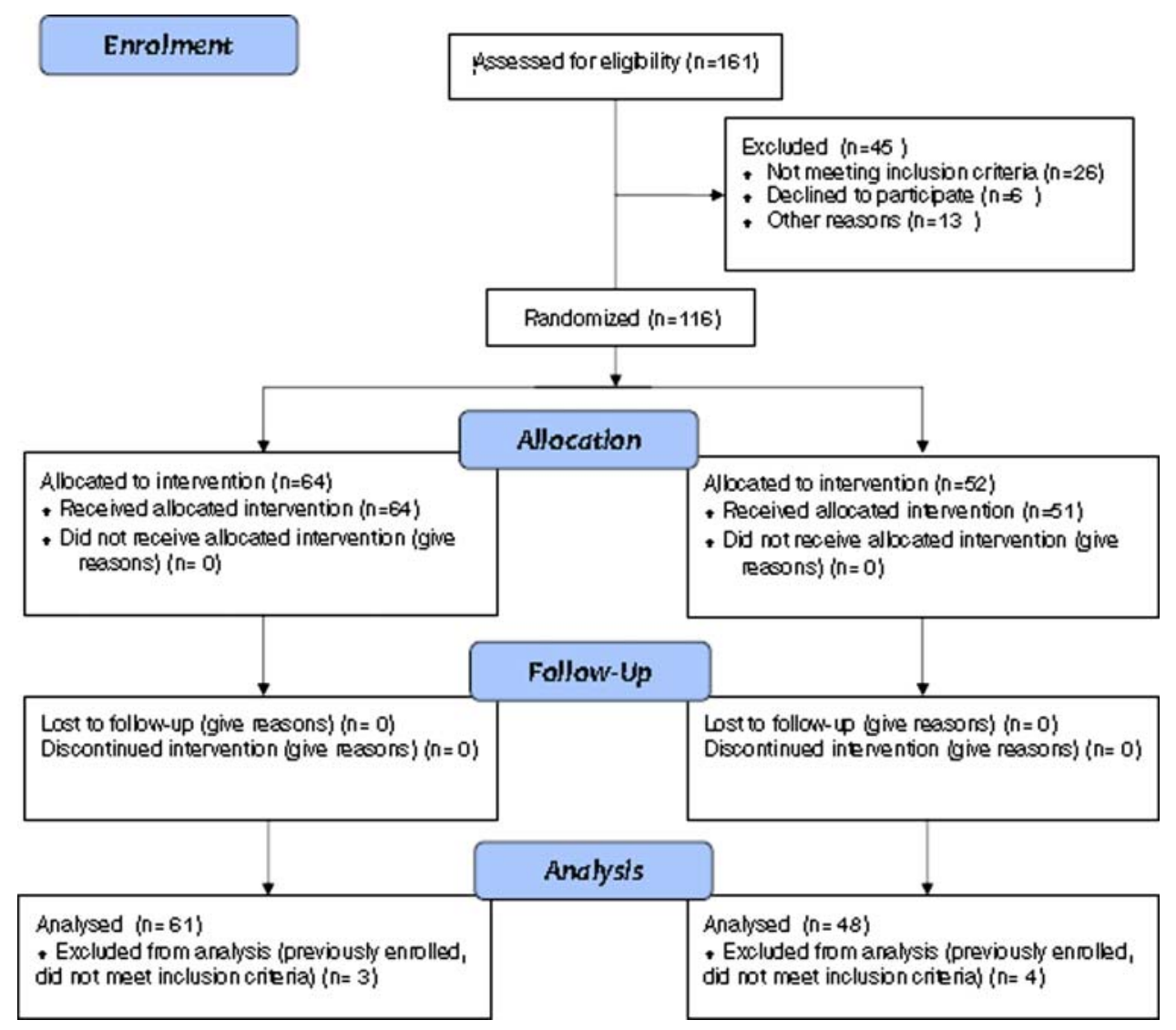

Figure 1 Consort trial profile. This figure is only reproduced in colour in the online version.

group and three in the magnesium group were inadvertently enrolled twice and the second presentation was excluded from the analysis (five events). Two other patients were excluded prior to analysis because of an inaccurate calculation of the percentage predicted $\mathrm{FEV}_{1}$ and initial failure to recognise pneumonia as the primary diagnosis.

Table 1 shows the baseline characteristics of the 109 patients included in the analysis. The denominator for some of the characteristics varies due to missing data for eight patients (five in the placebo group and three in the magnesium group). The mean age was 71 years (range 36-89); 53\% were men and 40\% were current smokers. Nearly $20 \%$ were on domiciliary oxygen and the average number of admissions in the last year was one. Mean (SD) $\mathrm{FEV}_{1}$ on arrival was 0.71 (0.25) 1 (range 0.15-1.43 1). The proportion of patients with an $\mathrm{FEV}_{1}$ on arrival of $>1000 \mathrm{ml}$ was $8 / 48(16.7 \%)$ in the magnesium group and $6 / 61(9.8 \%)$ in the placebo group.

For the primary outcome variable, $\mathrm{FEV}_{1}$ at $90 \mathrm{~min}(30 \mathrm{~min}$ after the third administration of the study drug), the mean (SD) $\mathrm{FEV}_{1}$ in the magnesium group $(\mathrm{N}=47)$ was $0.78(0.33) 1 \mathrm{com}-$ pared with $0.81(0.30) 1$ in the saline group $(\mathrm{N}=61)$, difference $-0.0261(95 \%$ CI -0.15 to $0.095, p=0.67)$. After adjustment for baseline $\mathrm{FEV}_{1}$ the difference was -0.0241 (95\% CI -0.07 to 0.026 ), $\mathrm{p}=0.34$ (see table 2).

For $\mathrm{FEV}_{1}$ at 30 and $60 \mathrm{~min}$, ANCOVA showed an adjusted difference of $-0.0431(95 \%$ CI -0.08 to $-0.009, \mathrm{p}=0.014)$ and $-0.0421(95 \% \mathrm{CI}-0.08$ to $-0.007, \mathrm{p}=0.02)$ respectively (ie, $\mathrm{FEV}_{1}$ was lower in the magnesium group after adjustment for baseline). No patients required NIV and there were no ICU admissions in either group. There were 43/48 (89.6\%) admissions to hospital in the magnesium group and 56/61 (91.8\%) in the saline group (RR of admission for magnesium vs placebo group $0.98,95 \%$ CI 0.86 to $1.10, \mathrm{p}=0.69$ ).

Reversibility (based on absolute change in $\mathrm{FEV}_{1}$ of at least $200 \mathrm{ml}$ and $>12 \%$ change from baseline using $90 \mathrm{~min}$ and baseline $\mathrm{FEV}_{1}$ ) by randomised group was as follows: $5 / 47(10.6 \%)$ in the magnesium group vs $6 / 61(9.8 \%)$ in the placebo group

Table 1 Baseline characteristics of patient group

\begin{tabular}{lll}
\hline & $\begin{array}{l}\text { Placebo } \\
(\mathrm{N}=61)\end{array}$ & $\begin{array}{l}\text { Magnesium } \\
(\mathrm{N}=48)\end{array}$ \\
\hline Mean (SD) age, years & $69.5(11.9)$ & $73.2(9.8)$ \\
Female sex, $\mathrm{n}(\%)$ & $30(49.2)$ & $21(43.8)$ \\
Current smokers, $\mathrm{n}(\%)$ & $22 / 56(39.3)$ & $18 / 45(40.0)$ \\
Mean (SD) amount smoked, pack-years & $45.0(30.7)$ & $41.3(21.3)$ \\
& $(\mathrm{N}=55)$ & $(\mathrm{N}=42)$ \\
Never smoker, $\mathrm{n}(\%)$ & $2 / 56(3.6)$ & $1 / 45(2.2)$ \\
Long term oral steroid use, $\mathrm{n}(\%)$ & $6 / 56(10.7)$ & $5 / 45(11.1)$ \\
Inhaled corticosteroid, $\mathrm{n}(\%)$ & $44 / 56(78.6)$ & $37 / 45(82.2)$ \\
Home nebuliser, $\mathrm{n}(\%)$ & $17 / 56(30.4)$ & $11 / 45(24.4)$ \\
Home oxygen, $\mathrm{n}(\%)$ & $10 / 56(17.9)$ & $8 / 45(17.8)$ \\
Diuretic use, $\mathrm{n}(\%)$ & $12 / 61(19.7)$ & $13 / 48(27.1)$ \\
Hospital admission in last year & $1.3(\mathrm{~N}=55)$ & $1.0(\mathrm{~N}=45)$ \\
Mean (SD) presentation $\mathrm{FEV}_{1}, \mathrm{I}$ & $0.72(0.25)$ & $0.69(0.26)$ \\
Mean (SD) presentation $\mathrm{FEV}_{1}, \%$ predicted & $29.7(9.2)$ & $28.2(9.3)$ \\
Mean (SD) baseline FEV $_{1}, \mathrm{l}$ & $0.74(0.28)$ & $0.74(0.28)$ \\
Serum magnesium level (mmol/l) & $0.78(0.10)$ & $0.81(0.08)$ \\
& $(\mathrm{N}=42)$ & $(\mathrm{N}=36)$ \\
\hline FEV ${ }_{1}$, forced expiratory volume in 1 s. & &
\end{tabular}




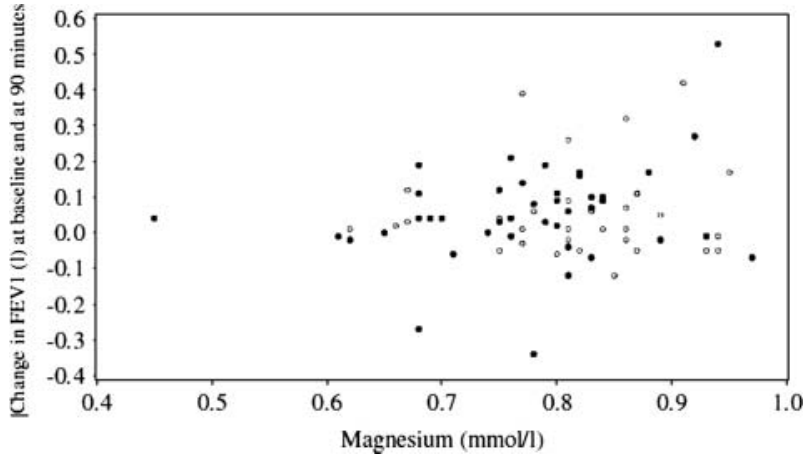

Figure 2 Change between forced expiratory volume in $1 \mathrm{~s}\left(\mathrm{FEV}_{1}\right)$ at baseline and at $90 \mathrm{~min}$ in relation to magnesium level (open symbols represent magnesium, closed symbols represent placebo).

(RR $1.08,0.35$ to $3.33, \mathrm{p}=0.89$ ). One participant in the magnesium group had missing 90 min $\mathrm{FEV}_{1}$ data.

Baseline serum magnesium levels ranged from 0.45 to $0.97 \mathrm{mmol} / \mathrm{l}$ (normal reference range $0.76-0.99 \mathrm{mmol} / \mathrm{l}$ ). There was no evidence that serum magnesium levels influenced the $\mathrm{FEV}_{1}$ response ( $\mathrm{p}$ for interaction $=0.51$ ), and the common correlation coefficient for the association between change in $\mathrm{FEV}_{1}$ at $90 \mathrm{~min}$ and time zero was $0.17(\mathrm{p}=0.13, \mathrm{~N}=77$ with complete data; figure 2).

No clinically significant adverse events were reported and no patients in either group needed additional bronchodilator therapy within the 90 min time period or were withdrawn because of clinical deterioration.

\section{DISCUSSION}

In this randomised double-blind placebo-controlled trial we were unable to demonstrate any clinical benefit with single or repeated administration of nebulised magnesium given as an adjunct to bronchodilator treatment on presentation to hospital with an AECOPD. In this study, the largest to date of nebulised magnesium in COPD, we were also unable to show any evidence of a relationship between serum magnesium, change in $\mathrm{FEV}_{1}$ and randomised treatment group.

Table 2 Serial forced expiratory volume in $1 \mathrm{~s}\left(\mathrm{FEV}_{1}\right)$ in patients receiving nebulised magnesium or placebo

\begin{tabular}{|c|c|}
\hline & Mean (SD) \\
\hline \multicolumn{2}{|l|}{$\mathrm{FEV}_{1}$ Time 0} \\
\hline Magnesium $(\mathrm{N}=48)$ & $0.74(0.28)$ \\
\hline Placebo $(\mathrm{N}=61)$ & $0.74(0.28)$ \\
\hline All $(\mathrm{N}=109)$ & $0.74(0.28)$ \\
\hline \multicolumn{2}{|l|}{$\mathrm{FEV}_{1}$ Time 30} \\
\hline Magnesium $(\mathrm{N}=48)$ & $0.74(0.29)$ \\
\hline Placebo $(\mathrm{N}=61)$ & $0.79(0.29)$ \\
\hline All $(\mathrm{N}=109)$ & $0.76(0.29)$ \\
\hline \multicolumn{2}{|l|}{$\mathrm{FEV}_{1}$ Time 60} \\
\hline Magnesium $(\mathrm{N}=48)$ & $0.76(0.31)$ \\
\hline Placebo $(\mathrm{N}=61)$ & $0.81(0.31)$ \\
\hline All $(\mathrm{N}=109)$ & $0.79(0.31)$ \\
\hline \multicolumn{2}{|l|}{$\mathrm{FEV}_{1}$ Time 90} \\
\hline Magnesium ( $N=47$ ) & $0.78(0.33)$ \\
\hline Placebo $(\mathrm{N}=61)$ & $0.81(0.30)$ \\
\hline All $(\mathrm{N}=108)$ & $0.79(0.31)$ \\
\hline
\end{tabular}

We considered several methodological issues in the design of the study that are relevant to its interpretation. The primary reason for including only subjects with an $\mathrm{FEV}_{1}<50 \%$ predicted (measured $20 \mathrm{~min}$ after commencement of initial salbutamol and ipratropium) was that, in groups with asthma, an effect with intravenous magnesium has been found in those with more severe disease. ${ }^{10} \mathrm{FEV}_{1}$ was chosen as the primary outcome variable as a sensitive, objective and repeatable measure of bronchodilator response in AECOPD. ${ }^{21} 22$ Specifically, the administration of a bronchodilator during AECOPD can increase the $\mathrm{FEV}_{1}$ and the FVC by $15-29 \%$ over a period of $60-120 \mathrm{~min}^{23}$

With regard to the protocol of bronchodilator administration, the reason for the initial salbutamol/ipratropium nebulisers at presentation was safety as well as ensuring some standardisation of bronchodilator treatment in the 20 min prior to randomisation. Guidelines for managing an AECOPD generally agree that bronchodilators are considered first-line treatment ${ }^{24-26}$ but may not recommend a specific dose. In stable COPD it has been shown that $88 \%$ of patients achieved $90 \%$ of maximal bronchodilation with doses of inhaled salbutamol $\leq 1.2 \mathrm{mg}$. ${ }^{27}$ It could be argued that this pre-dosing with bronchodilators meant patients had already reached their maximum bronchodilator response before administration of magnesium. Additionally, the combination of ipratropium and salbutamol is known to be superior to either agent alone. ${ }^{28} 29$ The magnesium used was formulated as an isotonic solution. This was important because both hypotonic and hypertonic nebuliser solutions can induce bronchoconstriction in patients with bronchial hyper-responsiveness. ${ }^{30}$

Recruitment proved difficult with 109 of a planned 200 patients studied, despite the extension of the study by 10 months. However, the study had equivalent power to that planned due to the smaller SD for $\mathrm{FEV}_{1}$ in the study compared with that used in the power calculations (based on our previous study of nebulised magnesium in acute severe asthma). ${ }^{13}$ This resulted in 95\% CIs that excluded the pre-nominated clinically important difference. The reason for the unequal numbers in the two groups was that the batches of magnesium and placebo provided by the hospital pharmacy were often incompletely used before expiring and a new batch being issued. Also, we recruited a group with severe airflow obstruction with a mean $\mathrm{FEV}_{1}$ of 0.741 .

The findings of this study raise a number of points for discussion. The first is the relation of this study to other studies of magnesium in COPD. There are only six placebo-controlled trials involving intravenous ${ }^{15-17} 19$ or nebulised ${ }^{18}$ magnesium or both ${ }^{20}$ in either acute exacerbations ${ }^{15} 161920$ or in the stable outpatient setting, ${ }^{17} 18$ and three are in abstract form only. ${ }^{15} 1819$

In two that involve intravenous magnesium in the setting of AECOPD, ${ }^{15} 161.2 \mathrm{~g}$ was administered following standard nebulised bronchodilator treatment. In their randomised doubleblind placebo-controlled trial of 24 subjects with an AECOPD, Hogg et $a l^{15}$ reported a significant reduction in the Modified Borg Dsypnoea Score $30 \mathrm{~min}$ after the start of the infusion (2.33 vs $1.08, \mathrm{p}<0.01)$ and a reduced length of inpatient stay in the intravenous magnesium group compared with placebo (4.27 vs 7.33 days, $p<0.05)$. In their randomised double-blind placebo-controlled trial of 72 subjects with AECOPD, Skorodin et $a l^{16}$ reported a significant increase in peak expiratory flow from initiation to 30 and $45 \mathrm{~min}$ later $(25.1$ vs $7.4 \mathrm{l} / \mathrm{min}$, $\mathrm{p}=0.03$ ) and a statistically non-significant trend towards a reduced need for hospitalisation with intravenous magnesium. Abreu Gonzalez et al administered either $1.5 \mathrm{~g}$ of magnesium (intravenous) or placebo on the first day and then vice versa on the other day in a randomised double-blind crossover design of 
24 patients. Salbutamol was administered $45 \mathrm{~min}$ after the placebo or magnesium. The mean increase in $\mathrm{FEV}_{1}$ was 0.181 compared with 0.0811 after placebo $(p=0.004)$. Interestingly, this bronchodilating effect was only observed after salbutamol administration. ${ }^{19}$

In a randomised double-blind placebo-controlled trial of 22 subjects with stable COPD, Do Amaral et $a l^{17}$ reported that an intravenous infusion of magnesium sulphate resulted in a significant reduction in lung hyperinflation measured as functional respiratory capacity $(-0.53$ vs $-0.051, p=0.04)$ and an increase in respiratory muscle strength measured as maximum inspiratory pressure $\left(6.9\right.$ vs $\left.-3.1 \mathrm{~cm} \mathrm{H}_{2} \mathrm{O}, \mathrm{p}=0.02\right)$. Together these studies indicate that a single intravenous dose of magnesium has clinical efficacy in both stable and acute exacerbations of COPD.

More recently, Nouira et al randomised 62 patients presenting to the ED with an AECOPD to receive either multiple nebulisations of terbutaline plus ipratropium or terbutaline plus a once-only bolus of intravenous magnesium ( $1.5 \mathrm{~g}$ ) followed by repeated nebulised terbutaline and nebulised magnesium (150 mg/nebule). There was no significant difference between the two groups in their primary outcome variable (which included hospital admission, intubation and hospital death rates). Patients given ipratropium (and terbutaline) had an average improvement in peak expiratory flow of $32 \mathrm{l} / \mathrm{min}(95 \%$ CI 19 to 43) compared with the magnesium group. Their reason for combining an intravenous bolus and repeated nebulisations was to ensure an adequate dose but to avoid potentially toxic blood levels. ${ }^{20}$

The only other study of nebulised magnesium investigated its effect in patients with stable COPD recruited from outpatients. The group that received magnesium as an adjunct to nebulised salbutamol showed a significant increase in $\mathrm{FEV}_{1}$ from 1.441 to $1.67 \mathrm{l}$ at $60 \mathrm{~min}(\mathrm{p}<0.05) .{ }^{18}$ However, this study does not appear to have been randomised or double-blind and was inadequately powered with only 18 patients studied. It is difficult to reach any conclusion about nebulised magnesium in COPD on the basis of this trial. We propose that our randomised doubleblind placebo-controlled design and greater power provide a higher level of evidence that nebulised magnesium as an adjunct to salbutamol treatment in the setting of AECOPD had no effect.

The second point is consideration of our findings in relation to the asthma literature. This is relevant because the reported efficacy of magnesium in asthma exacerbations is the pretext for its use in COPD. In asthma, magnesium has been administered both intravenously and via the nebulised route. The recent Cochrane review and meta-analysis of intravenous magnesium reported an improvement only in the severe subgroup in whom peak expiratory flow improved by $52.3 \mathrm{l} / \mathrm{min}$ (95\% CI 27 to 77.5 ) and $\mathrm{FEV}_{1}$ by $9.8 \%$ predicted (95\% CI $3.8 \%$ to $\left.15.8 \%\right) .{ }^{10}$ However, the Cochrane review of nebulised magnesium as an adjunct in acute asthma reported a non-significant improvement in pulmonary function in the nebulised magnesium group, but significant heterogeneity between trials precluded a definitive conclusion. ${ }^{14}$

This leads on to the third point, which concerns route of administration. Although individual trials in acute asthma, including our own, may show some benefit with nebulised magnesium, ${ }^{12} 13$ this was not conclusively shown in the meta-analysis ${ }^{14}$ and the efficacy of this route must remain in question. The benefits of repeated administration of nebulised magnesium include ease of administration with no need for an intravenous line. With regard to the dose of magnesium, this was based on the work in asthma. ${ }^{13}$
The fourth point for discussion, and related to the patient group, is that of the definition of asthma versus COPD and the spectrum of obstructive airways disease, particularly in the older age group. Increasingly, the complexity and heterogeneity of these conditions is recognised ${ }^{3132}$ and better definition of the patient group who may respond to magnesium may be crucial in clarifying its therapeutic effect.

The fifth point concerns the association between serum magnesium levels and COPD. ${ }^{33-35}$ Aziz et al retrospectively reviewed charts of 50 patients with stable COPD and 50 with an AECOPD. Those in the latter group had significantly lower magnesium levels. ${ }^{35}$ Bhatt et $a l^{34}$ also retrospectively reviewed magnesium levels and readmission rate and death in 100 patients with AECOPD. The sole predictor of frequent readmissions was low serum magnesium. Rolla et al measured magnesium levels in a group of 95 with severe but stable COPD; $11 \%$ had hypomagnesaemia and there was a significant inverse relationship between serum magnesium and the use of diuretics or length of oral steroid treatment. The authors concluded that serum magnesium should be routinely checked because of potential negative effects on respiratory muscle power. ${ }^{33}$ Earlier work has shown that correction of hypomagnesaemia is associated with improved respiratory muscle power. ${ }^{36}$ Interestingly, we did not find any relationship between serum magnesium, change in $\mathrm{FEV}_{1}$ and randomised treatment group. We are aware of one study that has characterised metallic elements in exhaled breath condensate and serum as novel biomarkers of exposure and susceptibility in AECOPD. Corradi et al found that exhaled magnesium levels were $50 \%$ higher in an exacerbation than at recovery (but there was no change in serum level). Manganese was the only other metallic element to show a similar pattern. ${ }^{37}$ The meaning of this is unclear; perhaps the high local magnesium levels in the airways explain why there is no great response to nebulised magnesium.

In conclusion, this randomised double-blind placebo-controlled trial has shown no evidence of efficacy of single or repeated nebulised magnesium as an adjunct to nebulised salbutamol in AECOPD. Our findings, together with previous studies, suggest that the priority for further investigation of magnesium in AECOPD should be with the intravenous route of administration.

Acknowledgements We thank staff at the emergency departments of Wellington and Hutt Hospitals New Zealand and the Pharmacy Staff at Wellington Hospital. Particular thanks are due to research fellows at the Medical Research Institute of New Zealand (who comprise the rest of the Magnesium COPD Study Team) who helped staff the roster. They are Dr Kyle Perrin, Dr Meme Wijesinghe, Dr Janine Pilcher, Dr Mitesh Patel, Dr James Fingleton, Dr Laird Cameron. Thanks are also due to the Medical Registrars at Wellington Hospital who also helped out on occasion.

Contributors The study was designed by RB, MW and PS. LE and PS coordinated the study and collected the data with $\mathrm{KW}, \mathrm{BH}$ and SJ. MW assisted with the statistical analysis. PS and RB wrote the report with help from the other authors.

Funding The study was funded by the Health Research Council of New Zealand. Competing interests None.

Ethics approval The trial was approved by the Central Regional Ethics Committee and written informed consent was obtained from all patients.

Provenance and peer review Not commissioned; internally peer reviewed.

\section{REFERENCES}

1 Fawcett W, Haxby E, Male D. Magnesium: physiology and pharmacology. Br J Anaesth 1999;83:302-20.

2 Herroeder S, Schonherr M, De Hert S, et al. Magnesium essentials for anesthiologists. Anesthesiology 2011;114:971-93.

3 Spivey W, Skobeloff E, Levin R. Effect of magnesium chloride on rabbit bronchial smooth muscle. Ann Emerg Med 1990;19:1107-12.

4 del Castillo J, Engbaek L. The nature of the neuromuscular block produced by magnesium. J Physiol 1954;124:370-84. 
5 Rosello H, Pla J. Sulfato de magnesio en la crisis de asma. Presna Med Argent 1936;23:1677-80.

6 Silverman $\mathrm{R}$, Osborne $\mathrm{H}$, Runge J, et al. IV magnesium sulfate in the treatment of acute severe asthma. Chest 2002;122:489-97.

7 Bloch $\mathrm{H}$, Silverman $\mathrm{R}$, Mancherje $\mathrm{N}$, et al. Intravenous magnesium sulphate as an adjunct in the treatment of acute asthma. Chest 1995;107:1576-81.

8 Skobeloff EM, Spivey WH, McNamara RM, et al. Intravenous magnesium sulphate for the treatment of acute asthma in the emergency department. JAMA 1989;262:1210-13.

9 Schenk P, Vonbank K, Schnack B, et al. Intravenous magnesium sulphate for bronchial hyperreactivity: a randomized, controlled, double-blind study. Clin Pharmacol Ther 2001;69:365-71.

10 Rowe B, Bretzlaff J, Bourdon C, et al. Magnesium sulphate for treating exacerbations of asthma in the emergency department. Cochrane Database Syst Rev 2006:3:1-26.

11 Mangat H, D'Souza G, Jacob M. Nebulized magnesium sulphate versus nebulized salbutamol in acute bronchial asthma: a clinical trial. Eur Respir J 1998:12:341-4.

12 Nannini L, Pendino J, Corna R, et al. Magnesium sulphate as a vehicle for nebulised salbutamol in acute asthma. Am J Med 2000;108:193-7.

13 Hughes R, Goldkorn A, Masoli M, et al. Use of isotonic nebulised magnesium sulphate as an adjuvant to salbutamol in the treatment of severe asthma in adults: randomised placebo-controlled trial. Lancet 2003:361:2114-17.

14 Blitz M, Blitz S, Beasley R, et al. Inhaled magnesium sulfate in the treatment of acute asthma. Cochrane Database Syst Rev 2006;3:1-28.

15 Hogg J, Mulrennan S, Everett C, et al. A single centre double-blind placebo-controlled pilot study into the use of intravenous magnesium sulphate for the treatment of acute exacerbation of COPD (abstract). Eur Respir J 2004; 24(Suppl 48):343s.

16 Skorodin M, Tenholder M, Yetter B, et al. Magnesium sulphate in exacerbations of chronic obstructive pulmonary disease. Arch Intern Med 1995;155:496-500.

17 Do Amaral AF, Rodrigues-Junior AL, Filho JT, et al. Effects of acute magnesium loading on pulmonary function of stable COPD patients. Med Sci Monit 2008;14:CR524-9.

18 Tagaya E, Tamaoki J, Kawatani K, et al. The effect of isotonic nebulized magnesium sulfate on pulmonary function in moderate to severe COPD. Am J Respir Crit Care Med 2004;169:A521.

19 Gonzalez J Abreu, Garcia C Hernandez, Gonzalez P Abreu, et al. Effect of magnesium sulphate on chronic obstructive pulmonary disease exacerbations requiring hospitalization: a randomised placebo-controlled trial (abstract). Arch Bronconeumol 2006;42:384-7.

20 Nouira S, Bouida W, Grissa M, et al. Magnesium sulphate versus ipratropium bromide in chronic obstructive pulmonary disease exacerabation: a randomised trial. Am J Ther Published Online First: 8 Mar 2012. doi:10.1097/MJT.0b013e3182459a8e
21 Niewoehner D, Collins D, Erbland M. Relation of FEV 1 to clinical outcomes during exacerbations of chronic obstructive pulmonary disease. Am J Respir Crit Care Med 2000;161:1201-5.

22 Tashkin D, Celli B, Decramer M, et al. Bronchodilator responsiveness in patients with COPD. Eur Respir J 2008:31:742-50.

23 Stoller J. Acute exacerbations of chronic obstructive pulmonary disease. N Engl J Med 2002;346:988-94.

24 Global Strategy for Chronic Obstructive Lung Disease (GOLD). http://www.goldcopd. org (accessed Dec 2011).

25 British Thoracic Society (BTS). BTS guidelines for the management of COPD. Thorax 1997;52:S1-28

26 National Institute for Clinical Excellence (NICE). Chronic obstructive pulmonary disease: national clinical guidelines for the management of COPD in adult sin primary and secondary care. Thorax 2004:59:51-232.

27 Teale C, Morrison J, Page R, et al. Dose response to inhaled salbutamol in chronic obstructive airways disease. Postgrad Med J 1991;67:754-6.

28 Combivent Inhalation Aerosol Study Group. In chronic obstructive pulmonary disease, a combination of ipratropium and albuterol is more effective than either agent alone. Chest 1994;105:1411-19.

29 Dorinsky $P$, Resiner $C$, Ferguson $G$, et al. The combination of ipratropium and albuterol optimizes pulmonary function reversibility testing in patients with COPD. Chest 1999;115:966-71.

30 Beasley R, Rafferty $\mathrm{P}$, Holgate $\mathrm{S}$. Adverse reactions to the non-drug constituents of nebuliser solutions. Br J Clin Pharmacol 1988;25:283-7.

31 Beasley $\mathrm{R}$, Weatherall $\mathrm{M}$, Travers J, et al. Time to define the disorders of the syndrome of COPD. Lancet 2009;374:670-2.

32 Diaz-Guzman E, Mannino D. Airway obstructive diseases in older adults: from detection to treatment. J Allergy Clin Immunol 2010;126:702-9.

33 Rolla G, Bucca C, Bugiani M, et al. Hypomagnesemia in chronic obstructive lung disease: effect of therapy. Magnesium Trace Elem 1990;9:132-6.

34 Bhatt $S$, Khandelwal $P$, Nanda $S$, et al. Serum magnesium is an independent predictor of frequent readmissions due to acute exacerbation of chronic obstructive pulmonary disease. Respir Med 2008;102:999-1003.

35 Azziz H, Blamoun A, Shubair M, et al. Serum magnesium levels and acute exacerbations of chronic obstructive pulmonary disease: a retrospective study. Ann Clin Lab Sci 2005:35:423-7.

36 Molloy D, Dhingra $S$, Solven $F$, et al. Hypomagnesemia and respiratory muscle power. Am Rev Respir Dis 1984;129:497-8.

37 Corradi M, Acampa O, Goldoni M, et al. Metallic elements in exhaled breath condensate and serum of patients with exacerbation of chronic obstructive pulmonary disease. Metallomics 2009;1:339-45. 